\title{
Left Ventricular Contraction Sequence in a Case Where the QRS Changed from Left to Atypical Right Bundle Branch Block
}

This article was published in the following Dove Press journal:

International Medical Case Reports Journal

\author{
Hideyuki Hara $\mathbb{D}^{\prime}$ \\ Takako Nagata ${ }^{2}$ \\ Hiroshi Ito' \\ Shinichi Niwano ${ }^{2}$ \\ Junya $\mathrm{Ako}^{2}$
}

'Division of Cardiology, Numazu City Hospital, Numazu, Japan; ${ }^{2}$ Department of Cardiovascular Medicine, Kitasato University School of Medicine,

Sagamihara, Japan
Correspondence: Hideyuki Hara Division of Cardiology, Numazu City Hospital, Aza-Harunoki 550, Higashi-Shiiji, Numazu City, Shizuoka Prefecture 4100302, Japan

Tel +8I-55-924-5I00

Fax +8I-55-924-5I33

Email hideyuki@med.kitasato-u.ac.jp

\begin{abstract}
A subgroup of right bundle branch block (RBBB) patients may exhibit a significant left ventricular (LV) activation delay. We evaluated echocardiography in a nonischemic heart failure patient whose QRS morphology changed from left bundle branch block (LBBB) to atypical RBBB. The septum to posterior wall motion delay (SPWMD) measured using the M-mode was $196 \mathrm{~ms}$ while the patient presented with LBBB but decreased to $32 \mathrm{~ms}$ after the morphology changed to RBBB. These changes were also associated with delayed appearance of the septal displacement peak. Speckle tracking longitudinal strain was evaluated using three standard apical views after the morphology changed to RBBB. The LV contraction initially appeared in the basal inferior wall and there was delayed anterior wall contraction. The LV contraction pattern in our patient changed when the QRS morphology changed to atypical RBBB. A specific LV contraction sequence observed in atypical RBBB may reflect a significant LV activation delay between the inferior and anterior wall.
\end{abstract}

Keywords: left bundle branch block, right bundle branch block, masquerading bundle branch block, bilateral bundle branch block, activation delay, longitudinal strain

\section{Introduction}

Left ventricular (LV) activation delay in heart failure (HF) patients with right bundle branch block (RBBB) is usually minor, when compared to that in patients with left bundle branch block (LBBB). However, a subgroup of RBBB patients with LBBB morphology in their limb leads have been described as masquerading $\mathrm{BBB}$ or bilateral $\mathrm{BBB}$, and are presumed to have a significant LV activation delay. ${ }^{1-3}$ We present a non-ischemic HF patient whose QRS morphology changed from LBBB to atypical RBBB. We evaluated his echocardiography before and after the QRS morphology changed and hypothesized the LV activation process in atypical RBBB.

\section{Case Report}

\section{Patient}

The patient was a 72-year-old man suffering from non-ischemic cardiomyopathy with a reduced $\mathrm{LV}$ ejection fraction of $25 \%$ to $30 \%$. He suffered from known, pre-existing LBBB with a QRS duration $>150 \mathrm{~ms}$ for 8 years. However, the QRS morphology in his chest leads changed to RBBB with progression of the left axis deviation (LAD) and 


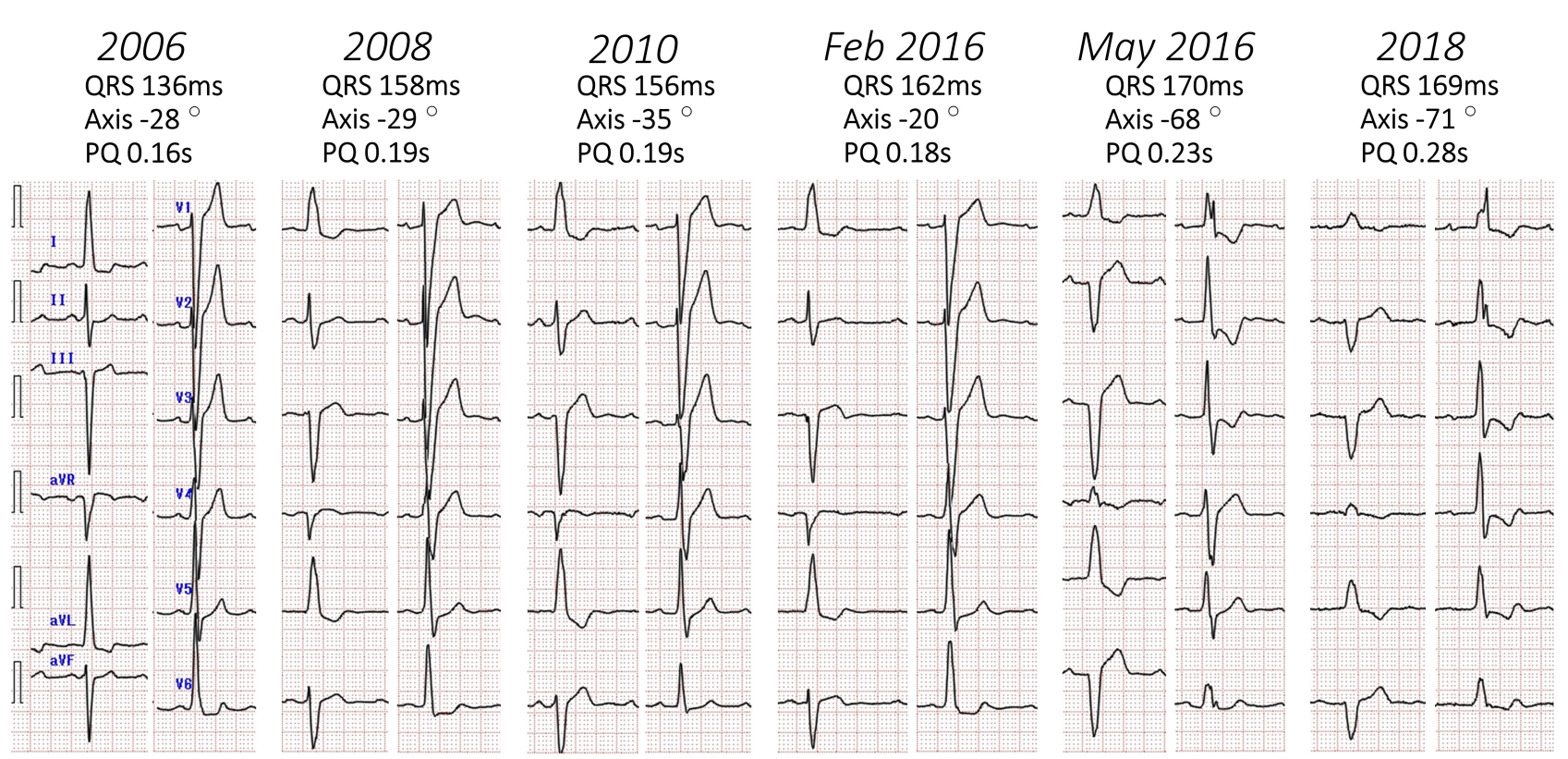

Figure I The patient's I2-lead electrocardiograms. He had LBBB with a QRS duration > I50 ms after 2008. The QRS morphology in his chest leads changed into RBBB in May 2016 with a progression of left axis deviation and prolongation of the PQ interval.

prolongation of the PQ interval (Figure 1). Six months after the QRS morphology changed, his New York Heart Association (NYHA) functional class worsened to III, and he was admitted to our hospital for HF treatment (Figure 2).

No significant luminal abnormalities were identified during coronary artery angiography.

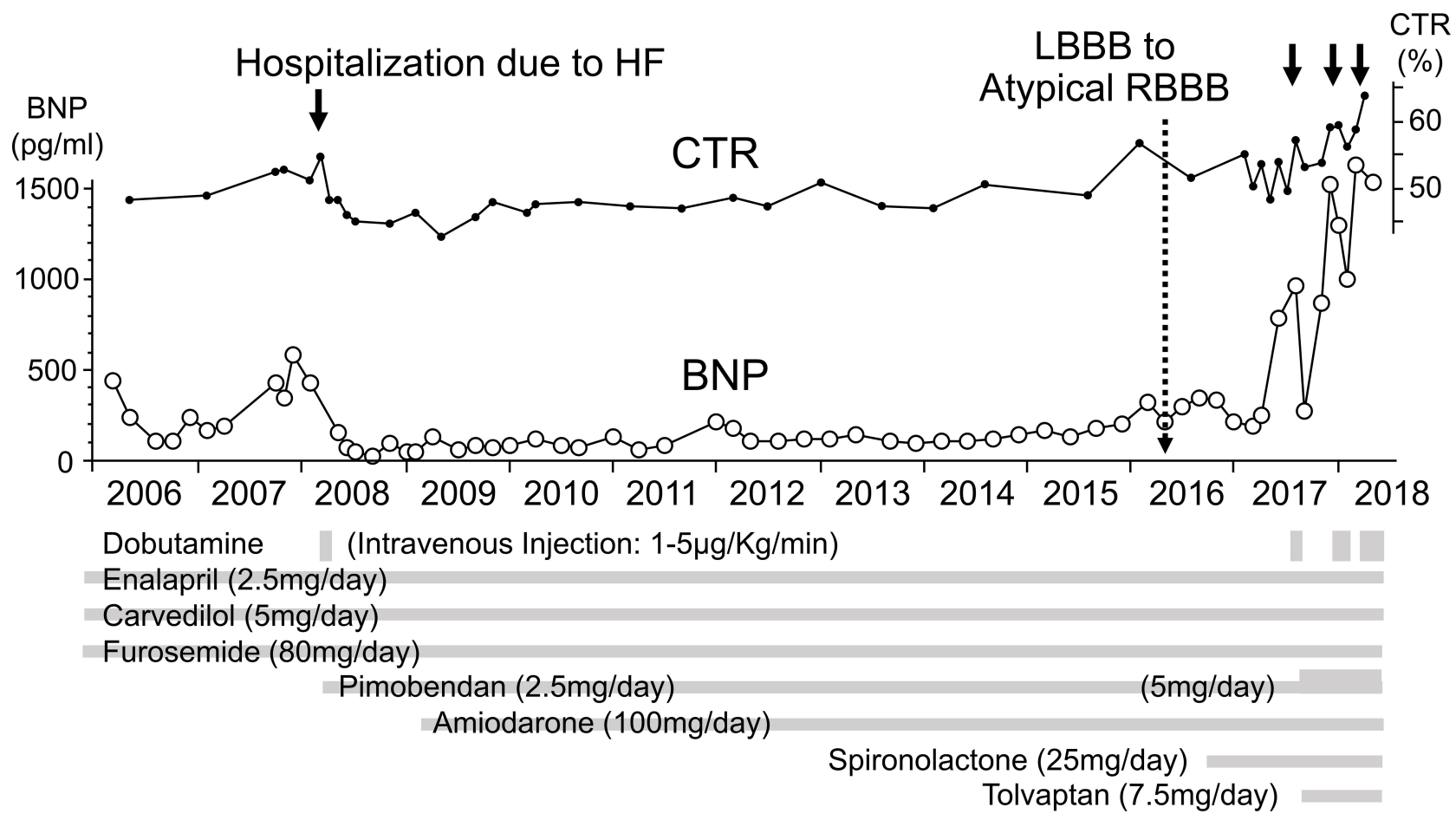

Figure 2 The clinical course. Hospitalization due to HF in 2008 (63 years). Treatment for non-ischemic cardiomyopathy (ejection fraction: $25-30 \%$ ) continued after 2008. Change in QRS morphology from LBBB to atypical RBBB in 2016 (7I years). Hospitalization due to HF deterioration in 2017 (72 years). Death due to HF in 2018 (73 years). Abbreviations: HF, heart failure; CTR, cardio-thoracic ratio; BNP, serum brain natriuretic peptide. 


\section{Echocardiography}

Two-dimensional (2D), pulsed Doppler and tissue Doppler echocardiography (Arietta system, Hitachi, Tokyo, Japan) were performed before and after the QRS morphology changed. Mechanical dyssynchrony was assessed using the following measurements: 1) LV and right ventricular (RV) pre-ejection period (PEP) using pulsed Doppler, ${ }^{4}$ and 2) septum to posterior wall motion delay (SPWMD) using M-mode. ${ }^{5}$ After RBBB onset, the LV-PEP did not change significantly (LBBB $180 \mathrm{~ms}$; RBBB: $190 \mathrm{~ms}$ ), but the RV-PEP was prolonged (LBBB $90 \mathrm{~ms}$; RBBB: 205 $\mathrm{ms})$. While the patient exhibited LBBB, the septum contracted earlier than the posterior wall and the SPWMD was 196 ms (Figure 3). After the onset of RBBB, the SPWMD decreased to $32 \mathrm{~ms}$ with late appearance of the septal displacement peak. Speckle tracking echocardiography was performed after RBBB onset using a GE Vivid E9 system (GE Vingmed Ultrasound, Horten, Norway). The LV longitudinal strain (LS) was evaluated using three standard apical views. Bulls-eye distribution maps of LS during the systolic period were constructed using off-line software (EchoPac, version 113, GE Vingmed Ultrasound). The LV contraction initially appeared in the basal inferior wall. The contracting area subsequently enlarged toward both the septum and lateral regions, and the anterior wall contracted late (Figure 4).

Standard diastolic filling parameters and mitral regurgitation (MR) were evaluated using the Arietta system (echo evaluations \#1 to \#3 in Table 1) and GE Vivid E9 system (\#4 and \#5). The MR severity was estimated by calculating the ratio of the MR jet area to the left atrium
(LA) area using the color Doppler. The severity was then graded as mild $(<20 \%)$, moderate $(20 \%$ to $40 \%)$, or severe $(>40 \%){ }^{6}$ After RBBB onset (\#3), the E wave velocity and $\mathrm{E} / \mathrm{A}$ ratio increased, the deceleration time (DT) of $\mathrm{E}$ wave shortened and the E/e' ratio increased, compared to the findings when the patient exhibited LBBB (\#1 and \#2). The MR severity worsened after RBBB onset. The E wave velocity, $\mathrm{E} / \mathrm{A}$ ratio and $\mathrm{DT}$ of $\mathrm{E}$ wave exhibited slight improvement during an evaluation on Mar 2017 (\#4), but worsened after a short while (\#5).

\section{Clinical Course}

Despite optimal medical treatment, which included an angiotensin-converting enzyme inhibitor, a beta blocker and diuretics, he was still classified as NYHA functional class III. Recently, several reports have suggested that RBBB patients with LBBB morphology in the limb leads have significant LV activation delays and respond better to cardiac resynchronization therapy (CRT) than those with typical RBBB. ${ }^{7,8}$ Therefore, we believe that our patient was a candidate for CRT. However, due to physical limitations in activities of daily living secondary to a prior accident, the patient refused CRT. Despite the fact that the patient maintained a sinus rhythm, and that the atrioventricular block did not progress beyond the first degree, he was hospitalized again within the year due to worsening HF. His ambulatory symptoms subsequently progressed to NYHA functional class IV. Echocardiography revealed a dilated LV chamber and persistent, severe mitral regurgitation (MR). He ultimately died two years after RBBB onset.
A

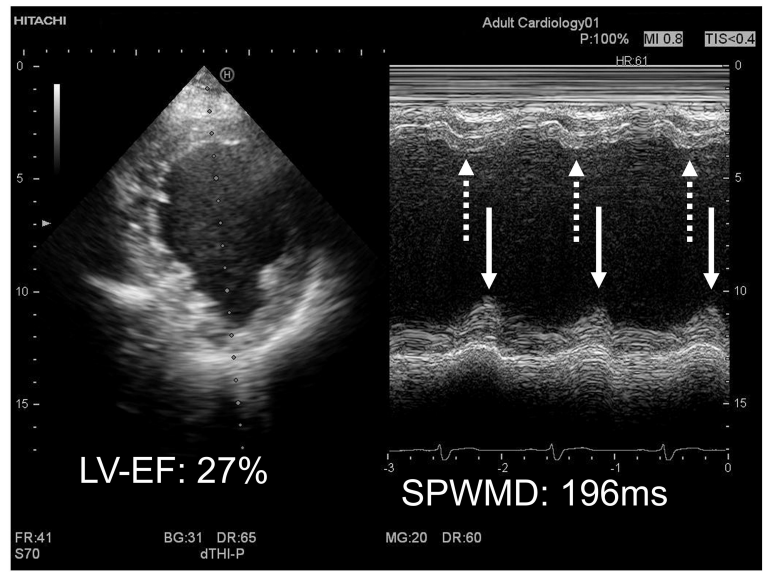

B

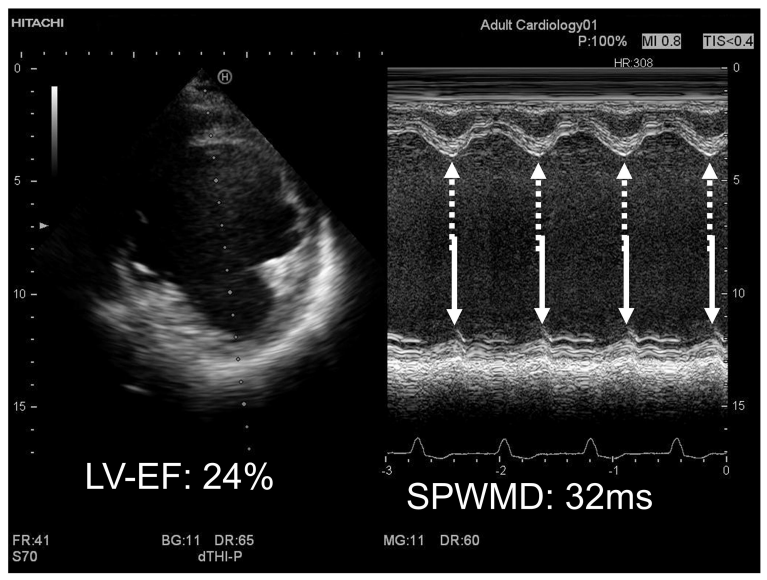

Figure 3 (A) M-mode image in the mid-LV short axis view recorded in April 2015 (at the time of LBBB). (B) M-mode image recorded in May 2016 (at the time of atypical RBBB). Arrows indicate the displacement peak of the anteroseptal (dotted arrow) and posterior (inferolateral) wall (solid arrow).

Abbreviations: LV-EF, left ventricular ejection fraction; SPWMD, septum to posterior wall motion delay. 

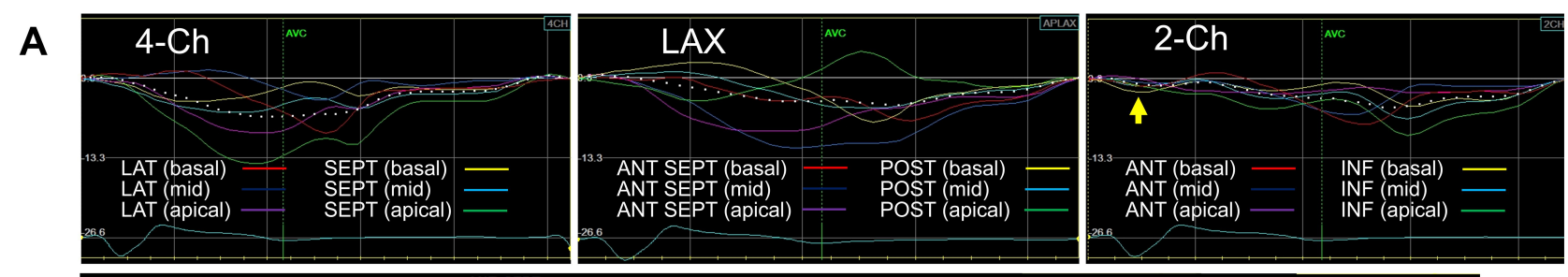

B

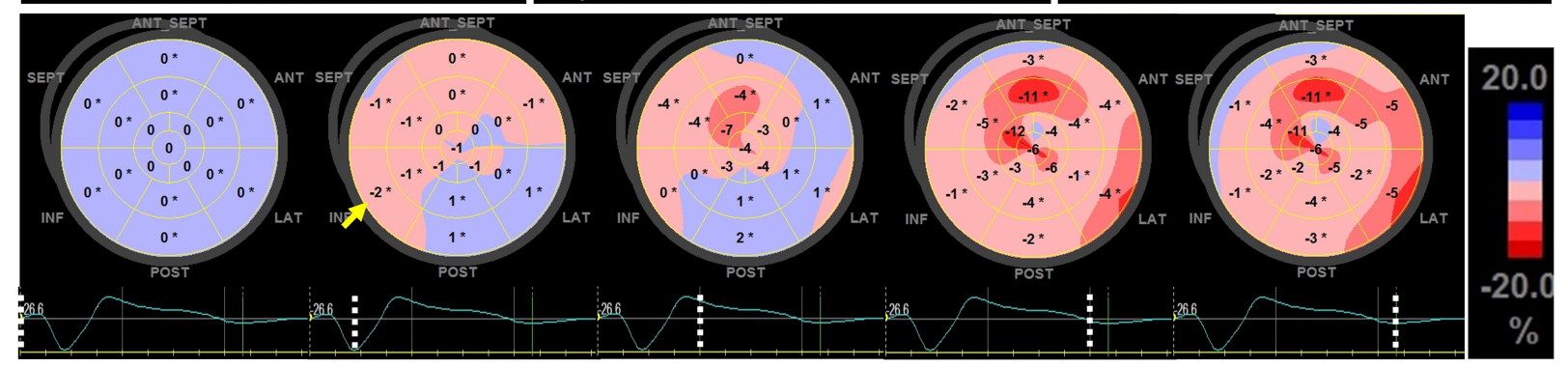

Figure 4 Speckle tracking longitudinal strain (LS) evaluations performed in Mar 2017 (at the time of atypical RBBB). (A) Segmental LS curves in three standard apical views. The yellow arrow indicates the peak of negative strain which appeared within the QRS duration in the basal inferior wall. (B) Bulls-eye maps demonstrating temporal changes in regional LS distribution during the systolic period (QRS onset to aortic valve closing). Negative strain initially appeared in the basal inferior wall (yellow arrow). The contracting area enlarged toward both the septum and lateral regions, and the anterior wall contracted late. The dotted line on the electrocardiogram indicates the time phase of each bulls-eye display.

\section{Discussion}

\section{Ventricular Activation in Atypical RBBB}

Ventricular conduction disorders with RBBB morphology in the chest leads and LBBB morphology in the limb leads (ie, LAD and absent S-wave in leads I-aVL) may be associated with significantly delayed LV activation. Evaluations of ventricular endocardial activation sequences using threedimensional electro-anatomical mapping demonstrated that these atypical RBBB patients have a biventricular activation delay. ${ }^{9}, 10$ In these reports, ventricular activation started in the inferior wall or lower septum of the LV and propagated toward the LV anterior or anterolateral wall. Altered ventricular activation sequences in atypical RBBB are believed to be inevitably accompanied by mechanical dyssynchrony. During the evaluations of LS using speckle tracking echocardiography, an early systolic LV contraction was observed in the inferior wall, followed by late contraction in the anterior wall. ${ }^{7,11}$

There are a few reports of LBBB patients whose QRS morphology changed to atypical RBBB., ${ }^{2,3,12-14}$

Table I Echocardiographic Parameters Before and After the QRS Morphology Changed

\begin{tabular}{|c|c|c|c|c|c|}
\hline \multirow[t]{3}{*}{ Echo Evaluation } & \#I & \#2 & \#3 & \#4 & \#5 \\
\hline & 2015 & 2015 & 2016 & 2017 & 2017 \\
\hline & Apr & Nov & May & Mar & Jun \\
\hline QRS morphology & LBBB & LBBB & RBBB & RBBB & RBBB \\
\hline LV-EF (\%) & 27 & 27 & 24 & 25 & 28 \\
\hline LVIDd (mm) & 76 & 76 & 75 & 82 & 82 \\
\hline LA dimension $(\mathrm{mm})$ & 40 & 42 & 45 & 43 & 42 \\
\hline E wave velocity $(\mathrm{cm} / \mathrm{s})$ & 37 & 36 & 85 & 55 & 109 \\
\hline A wave velocity $(\mathrm{cm} / \mathrm{s})$ & 71 & 64 & 51 & 61 & 67 \\
\hline E/A ratio & 0.5 & 0.6 & 1.7 & 0.9 & 1.6 \\
\hline DT of E wave (ms) & 250 & 263 & 117 & 200 & 103 \\
\hline Septal e' wave $(\mathrm{cm} / \mathrm{s})$ & 4.7 & 4.9 & 7.3 & 4.5 & 4.8 \\
\hline E/e' ratio & 7.9 & 7.4 & 11.6 & 12.3 & 22.6 \\
\hline Mitral regurgitation & moderate & moderate & severe & severe & severe \\
\hline
\end{tabular}

Abbreviations: LBBB, left bundle branch block; RBBB, right bundle branch block; LV-EF, left ventricular ejection fraction; LVIDd, left ventricular internal dimension in diastole; LA, left atrium; DT, deceleration time. 
Tzogias et al evaluated the QRS morphology in patients who developed transient RBBB during right heart catheterization. ${ }^{12}$ Patients with a normal QRS complex, left anterior fascicular block, or left posterior fascicular block at baseline developed a typical RBBB pattern with an S-wave in leads I-aVL. On the other hand, patients with LBBB at baseline exhibited LAD and 7 out of 11 patients developed atypical RBBB with no S-wave in leads I-aVL. Wu et al performed atrial pacing in patients who had manifested periods of both LBBB and RBBB, and recorded a His bundle electrogram, as well as a 12-lead electrocardiogram. ${ }^{13}$ The $\mathrm{H}-\mathrm{V}$ interval was prolonged and LAD progressed in the patients with LBBB associated with ratedependent RBBB. The authors presumed that there was a critical left bundle conduction delay in these LBBB patients, but that the block was incomplete due to residual conduction from the left posterior branch. From these reports, RBBB patients with LAD and an absent $\mathrm{S}$-wave in leads $1-\mathrm{aVL}$ are thought to have a significant LV activation delay comparable to that in patients with LBBB. A more definitive LAD was usually observed in conjunction with the change from LBBB to atypical RBBB. ${ }^{3,12,13}$ This may indicate that activation in atypical $\mathrm{RBBB}$ propagates from a more inferior region, when compared to LBBB.

\section{Criteria for Complete LBBB}

The electrocardiograms from 2008 to February 2016 in our patient satisfied the conventional criteria for complete LBBB (Figure 1): QRS duration $\geq 120 \mathrm{~ms}$; QS or $\mathrm{rS}$ in lead $\mathrm{V} 1$; broad $\mathrm{R}$ waves in leads $\mathrm{I}, \mathrm{aVL}, \mathrm{V} 5$, or V6; and absent Q waves in leads V5 and V6 ${ }^{15}$ These criteria are usually used to diagnose LBBB in patients who may be CRT candidates. ${ }^{16}$ However, the ST and $\mathrm{T}$ waves were not apposite in leads V4 to V6, and the presence of mid-QRS notching/slurring was not clearly present in more than two leads among leads I, aVL, V1, V2, V5, and V6. Our patient therefore did not satisfy the stricter criteria for complete LBBB. ${ }^{17,18}$ Patients who satisfy the conventional criteria for complete LBBB may exhibit conduction via the residual left bundle branch. ${ }^{9,19-21}$ Atrioventricular conduction was preserved after RBBB onset, likely indicating conduction between the His bundle and LV at the time of LBBB.

\section{Differences in LV Contraction in LBBB and Atypical RBBB}

The LV contraction pattern changed before and after the QRS morphology changed (Figure 3 and Supplementary $\underline{\text { Videos S1}}, \underline{\mathrm{S}}, \underline{\mathrm{S} 3}$, and $\underline{\mathrm{S}}$ ). As far as we know, this is the first report to evaluate the change in LV contraction patterns in conjunction with the change from LBBB to atypical RBBB. The septum in our patient contracted earlier than the posterior wall when the patient exhibited LBBB. After the change to atypical RBBB, the septum contracted later, and the SPWMD was reduced. On the other hand, the LV contraction initially appeared in the basal inferior wall (Figure 4 and Supplementary Video S5). Pre-ejection inward movement of the septum in patients with LBBB was considered to be active contraction due to the septal activation..$^{22}$ Presumably, at the time of LBBB, our patient had a significant left-sided conduction block, yet conduction via the left posterior branch was preserved. The LV was thought to be activated via signals from right-to-left transseptal conduction and the left posterior branch. After RBBB onset, the LV was only activated via the left posterior branch, which resulted in LV contraction initiated from the inferior region and the progression of LAD.

There are concerns of heart block onset in patients with atypical $\mathrm{RBBB}^{23}$ These patients usually have severe underlying heart disease and may develop $\mathrm{HF}^{24} \mathrm{HF}$ in our patient worsened after the QRS morphology changed to atypical RBBB and it became progressively more difficult to manage HF pharmaceutically (Figure 2). We believe that patients with atypical RBBB and symptoms of HF should be considered for early treatment, such as CRT, to ensure optimal timing of device implantation.

\section{Conclusions}

The LV contraction pattern in a patient with LBBB changed when the QRS morphology changed to atypical RBBB. The specific LV contraction sequence observed in atypical RBBB is thought to reflect a significant LV activation delay between the inferior and anterior wall.

\section{Consent for Publication}

Written informed consent was obtained from the patient's family for this case presentation. Our institutional approval is not required to publish the case details. 


\section{Funding}

There is no funding to report.

\section{Disclosure}

The authors report no conflicts of interest for this work.

\section{References}

1. Rosenbaum MB. Types of right bundles branch block and their clinical significance. J Electrocardiol. 1968;1(2):221-232.

2. Unger PN, Lesser ME, Kugel VH, Lev M. The concept of masquerading bundle-branch block; an electrocardiographic-pathologic correlation. Circulation. 1958;17(3):397-409. doi:10.1161/01. CIR.17.3.397

3. Richman JL, Wolff L. Left bundle branch block masquerading as right bundle branch block. Am Heart J. 1954;47(3):383-393. doi:10.1016/0002-8703(54)90295-1

4. Gorcsan J 3rd, Abraham T, Agler DA, et al. Echocardiography for cardiac resynchronization therapy: recommendations for performance and reporting-a report from the American Society of echocardiography dyssynchrony writing group endorsed by the heart rhythm society. J Am Soc Echocardiogr. 2008;21(3):191-213. doi:10.1016/ j.echo.2008.01.003

5. Pitzalis MV, Iacoviello M, Romito R, et al. Ventricular asynchrony predicts a better outcome in patients with chronic heart failure receiving cardiac resynchronization therapy. $\mathrm{J} \mathrm{Am} \mathrm{Coll} \mathrm{Cardiol.} \mathrm{2005;45}$ (1):65-69. doi:10.1016/j.jacc.2004.09.058

6. Zoghbi WA, Enriquez-Sarano M, Foster E, et al. Recommendations for evaluation of the severity of native valvular regurgitation with two-dimensional and Doppler echocardiography. $J \quad A m$ Soc Echocardiogr. 2003;16(7):777-802. doi:10.1016/S0894-7317(03) 00335-3

7. Atwater BD, Wagner GS, Kisslo J, Risum N. The electromechanical substrate for response to cardiac resynchronization therapy in patients with right bundle branch block. Pacing Clin Electrophysiol. 2017;40 (12):1358-1367. doi:10.1111/pace.13231

8. Pastore G, Morani G, Maines M, et al. Patients with right bundle branch block and concomitant delayed left ventricular activation respond to cardiac resynchronization therapy. Europace. 2018;20 (11):e171-e178. doi:10.1093/europace/eux362

9. Peichl P, Kautzner J, Cihak R, Bytesnik J. The spectrum of inter- and intraventricular conduction abnormalities in patients eligible for cardiac resynchronization therapy. Pacing Clin Electrophysiol. 2004;27 (8):1105-1112. doi:10.1111/j.1540-8159.2004.00592.x

10. Fantoni C, Kawabata M, Massaro R, et al. Right and left ventricular activation sequence in patients with heart failure and right bundle branch block: a detailed analysis using three-dimensional non-fluoroscopic electroanatomic mapping system. J Cardiovasc Electrophysiol. 2005;16(2):112-119; discussion 120-111. doi:10.1046/j.1540-8167.2005.40777.x

11. Leeters IP, Davis A, Zusterzeel R, et al. Left ventricular regional contraction abnormalities by echocardiographic speckle tracking in combined right bundle branch with left anterior fascicular block compared to left bundle branch block. J Electrocardiol. 2016;49 (3):353-361. doi:10.1016/j.jelectrocard.2016.02.002
12. Tzogias L, Steinberg LA, Williams AJ, et al. Electrocardiographic features and prevalence of bilateral bundle-branch delay. Circ Arrhythm Electrophysiol. 2014;7(4):640-644. doi:10.1161/ CIRCEP.113.000999

13. Wu D, Denes P, Dhingra RC, et al. Electrophysiological and clinical observations in patients with alternating bundle branch block. Circulation. 1976;53(3):456-464. doi:10.1161/01.CIR.53.3.456

14. Padanilam BJ, Morris KE, Olson JA, et al. The surface electrocardiogram predicts risk of heart block during right heart catheterization in patients with preexisting left bundle branch block: implications for the definition of complete left bundle branch block. $J$ Cardiovasc Electrophysiol. 2010;21(7):781-785.

15. Brignole M, Auricchio A, Baron-Esquivias G, et al. 2013 ESC Guidelines on cardiac pacing and cardiac resynchronization therapy: the task force on cardiac pacing and resynchronization therapy of the European Society of Cardiology (ESC). Developed in collaboration with the European Heart Rhythm Association (EHRA). Eur Heart J. 2013;34(29):2281-2329.

16. Zareba W, Klein H, Cygankiewicz I, et al. Effectiveness of cardiac resynchronization therapy by QRS Morphology in the Multicenter Automatic Defibrillator Implantation Trial-Cardiac Resynchronization Therapy (MADIT-CRT). Circulation. 2011;123 (10):1061-1072. doi:10.1161/CIRCULATIONAHA.110.960898

17. Surawicz B, Childers R, Deal BJ, et al. AHA/ACCF/HRS recommendations for the standardization and interpretation of the electrocardiogram: part III: intraventricular conduction disturbances: a scientific statement from the American Heart Association Electrocardiography and Arrhythmias Committee, Council on Clinical Cardiology; the American College of Cardiology Foundation; and the Heart Rhythm Society: endorsed by the International Society for Computerized Electrocardiology. Circulation. 2009;119(10):e235-240.

18. Strauss DG, Selvester RH, Wagner GS. Defining left bundle branch block in the era of cardiac resynchronization therapy. Am J Cardiol. 2011;107(6):927-934. doi:10.1016/j.amjcard.2010.11.010

19. Vassallo JA, Cassidy DM, Marchlinski FE, et al. Endocardial activation of left bundle branch block. Circulation. 1984;69(5):914-923. doi:10.1161/01.CIR.69.5.914

20. Rodriguez LM, Timmermans C, Nabar A, Beatty G, Wellens HJ. Variable patterns of septal activation in patients with left bundle branch block and heart failure. $J$ Cardiovasc Electrophysiol. 2003;14(2):135-141. doi:10.1046/j.1540-8167.2003.02421.x

21. Auricchio A, Fantoni C, Regoli F, et al. Characterization of left ventricular activation in patients with heart failure and left bundle-branch block. Circulation. 2004;109(9):1133-1139. doi:10.1161/01.CIR.0000118502.91105.F6

22. Gjesdal O, Remme EW, Opdahl A, et al. Mechanisms of abnormal systolic motion of the interventricular septum during left bundle-branch block. Circ Cardiovasc Imaging. 2011;4(3):264-273. doi:10.1161/CIRCIMAGING.110.961417

23. Kaimoto S, Kawasaki T, Taniguchi T, Kawasaki S, Kamitani T, Sugihara H. Masquerading bundle branch block as a marker of poor prognosis. J Cardiol Cases. 2013;8(1):e57-e59. doi:10.1016/j. jccase.2013.04.005

24. Akinyele OA, Balogun MO, Adebayo RA, et al. Masquerading bundle branch block in a patient with heart failure in Nigeria. Int Med Case Rep J. 2020;13:105-110. doi:10.2147/IMCRJ.S237077 


\section{Publish your work in this journal}

The International Medical Case Reports Journal is an international, peer-reviewed open-access journal publishing original case reports from all medical specialties. Previously unpublished medical posters are also accepted relating to any area of clinical or preclinica science. Submissions should not normally exceed 2,000 words or 4 published pages including figures, diagrams and references. The manuscript management system is completely online and includes a very quick and fair peer-review system, which is all easy to use. Visit http://www.dovepress.com/testimonials.php to read real quotes from published authors. 